\title{
A Supramolecular Model for the Co-Catalytic Role of Nitro Compounds in Brønsted Acid Catalyzed Reactions
}

\author{
Joel J. Montalvo-Acosta, ${ }^{[a]}$ Marian Dryzhakov ${ }^{[b]}$ Edward Richmond ${ }^{[b]}$ Marco Cecchini, ${ }^{\text {[a] }}$ and \\ Joseph Moran*[b]
}

\begin{abstract}
Nitro compounds are known to change reaction rates and kinetic concentration dependence of Brønstedacid-catalyzed reactions. Yet, no mechanistic model exists to account for these observations. In this work, an atomistic model for the catalytically active form for an alcohol dehydroazidation reaction is presented, which is generated by DFT calculations and consists of an $\mathrm{H}$-bonded aggregate of two molecules of Brønsted acid and two molecules of nitro compound. The computed $\mathrm{O}-\mathrm{H}$ stretching frequencies for the aggregate indicate they are stronger acids than the individual acid molecules and serve as predictors for experimental reaction rates. By applying the model to a chemically diverse set of potential promoters, it was predicted and verified experimentally that sulfate esters induce a similar co-catalytic effect. The important implication is that Brønsted-acid catalysis must be viewed from a supramolecular perspective that accounts for not only the $\mathrm{p} K_{\mathrm{a}}$ of the acid and the bulk properties of a solvent, but also the weak interactions between all molecules in solution.
\end{abstract}

The proton provides the most common and important way to catalyze organic reactions, ${ }^{[1]}$ however it need not always operate in isolation. Within a single molecular catalyst, such as in the active site of an enzyme, multiple hydrogen-bond donors (or Brønsted acidic sites) can work in cooperation to increase the overall acidity and hence catalytic activity. ${ }^{[2]}$ Cooperative $\mathrm{H}$ bonding and Brønsted acidity is also possible between molecules, when multiple $\mathrm{H}$-bond donors or Brønsted acid molecules interact to generate an aggregate that is a more effective catalyst than either individual molecule. ${ }^{[3]}$ However, what role

[a] Dr. J. J. Montalvo-Acosta, Dr. M. Cecchini

Université de Strasbourg, CNRS, UMR 7177

67000 Strasbourg (France)

E-mail: mcecchini@unistra.fr

[b] Dr. M. Dryzhakov, Dr. E. Richmond, Prof. Dr. J. Moran

Université de Strasbourg, CNRS, ISIS UMR 7006

67000 Strasbourg (France)

E-mail:moran@unistra.fr

$\square$ Supporting information and the ORCID identification number(s) for the

iD author(s) of this article can be found under:

https://doi.org/10.1002/chem.202000368.

Part of a Special Issue for the 8th EuChems Chemistry Congress consisting of contributions from selected speakers and conveners. To view the complete issue, visit Issue 48 the bystander molecules-those that are not $\mathrm{H}$-bond donors or Brønsted acids-might play, if any, remains to be elucidated. Though poorly understood, a few papers indicate that interactions between Brønsted acids and seemingly innocuous solvents or additives, particularly nitro compounds, can have a profound influence on reactivity both in terms of accelerating reaction rates and by changing the concentration dependence of the reaction. In the 1960s, Pocker and co-workers found that the hydrochlorination of olefins was not only markedly faster when carried out in nitromethane compared to other solvents, but also displayed an atypical second order kinetic concentration dependence on $\mathrm{HCl}^{[4]}$ Further investigations to uncover why this effect would be exclusive to nitromethane were not made. ${ }^{[5]}$ In 2015 , some of us observed that catalytic quantities of nitro compounds in benzene were highly specific to accelerate the dehydroazidation of tertiary aliphatic alcohols catalyzed by $\mathrm{B}\left(\mathrm{C}_{6} \mathrm{~F}_{5}\right)_{3} \cdot \mathrm{H}_{2} \mathrm{O}(\mathrm{BCF})^{[6]}$ a strong Brønsted acid of comparable strength to $\mathrm{HCl}^{[7]}$ The reaction was found to display a second order concentration dependence with respect to the nitro compound as well as a second order concentration dependence with respect to BCF. In contrast, when carried out in the absence of nitro compounds, the reaction was much slower and found to be first order with respect to BCF (Figure 1). Snyder's group has also observed Brønsted acid-catalyzed reactivity that is highly specific to wet nitromethane. ${ }^{[8]}$ No model currently exists to account for why the presence of a nitro compound, sometimes present only in catalytic quantities, could change the concentration dependence and rate of at least these two different Brønsted acid catalyzed reactions. Here, using DFT calculations and vibrational analysis, we obtain a structural model for a higher order aggregate formed by nitro compounds and Brønsted acids, examine the effect of aggregation on acidity, show that the model can be used to predict reaction rates for a set of new nitro compounds in alcohol dehydroazidation, and even predict a new class of "template" molecules that can induce a similar co-catalytic effect. Beyond the specific reaction in question, the present results imply that a comprehensive understanding of Brønsted acid catalyzed reactions can only be achieved by taking into account the weak interactions between the Brønsted acids and all molecules in solution.

The dehydroazidation of tertiary alcohols exhibits a secondorder kinetic dependence on the concentration of BCF and a second-order kinetic dependence on nitro compound ${ }^{[6]}$ As there exists significant spectroscopic and catalytic evidence that nitro compounds can act as $\mathrm{H}$-bond acceptors, ${ }^{[9]}$ we 
A Hydrochlorination of olefins (Ref. [4])

$$
\begin{aligned}
& \underbrace{\mathrm{R}^{3}}_{\mathrm{R}^{2}} \stackrel{\mathrm{HCl}}{\mathrm{R}_{\text {solvent }}} \\
& \text { solvent rate } \\
& \text { toluene, THF }[\mathrm{HCl}] \\
& \mathrm{MeNO}_{2} \quad[\mathrm{HCl}]^{2}
\end{aligned}
$$

B Dehydroazidation of tertiary alcohols (Ref. [6])

$$
\begin{aligned}
& \underset{\mathrm{R}_{1}}{\overbrace{\mathrm{R}_{2}}^{\mathrm{OH}}} \stackrel{\substack{\mathrm{B}\left(\mathrm{C}_{6} \mathrm{~F}_{5}\right)_{3} \cdot \mathrm{H}_{2} \mathrm{O} \text { (cat.) } \\
\mathrm{TMSN}_{3}(3 \text { equiv) }}}{\mathrm{C}_{6} \mathrm{H}_{6,23^{\circ} \mathrm{C}}} \\
& \underline{\text { additive } \quad \underline{\text { rate }}} \\
& \text { none } \quad\left[\mathrm{B}\left(\mathrm{C}_{6} \mathrm{~F}_{5}\right)_{3} \cdot \mathrm{H}_{2} \mathrm{O}\right] \text {; slow } \\
& \mathrm{MeNO}_{2} \quad\left[\mathrm{~B}\left(\mathrm{C}_{6} \mathrm{~F}_{5}\right)_{3} \cdot \mathrm{H}_{2} \mathrm{O}\right]^{2} ;\left[\mathrm{MeNO}_{2}\right]^{2} \text {; fast }
\end{aligned}
$$

Why is the kinetic concentration dependence influenced by $\mathrm{MeNO}_{2}$ ?

Figure 1. The effect of nitromethane on the kinetic concentration dependence of reactions consuming (A) or catalyzed (B) by Brønsted acids.

postulated that the reaction kinetics could be explained by the intermediacy of a catalytic competent aggregate involving $\mathrm{H}$ bonded self-assembly of two BCF with two nitro-compound molecules. Structural models of the tetrameric (2:2) assembly of BCF with nitromethane were generated by connecting the molecules through pairs of $\mathrm{H}$-bonds and optimizing the geometry of the aggregate by DFT. Given the complexity of the configurational space to be explored, an initial conformational search was carried out using the HF-3c semi-empirical method ${ }^{[10]}$ then refined by DFT calculations using the $\omega B 97 X-D$ functional ${ }^{[11]}$ with the $6-31 \mathrm{G}(\mathrm{d}, \mathrm{p})$ basis set; see Supporting Information for details. DFT optimization results in an almost flat, rectangular hydrogen-bonded network formed between the nitro groups of two nitromethanes and two boron hydrates (Figure 2). The tetrameric arrangement is stabilized by two sets of non-equivalent $\mathrm{H}$-bonds, two shorter and two longer. Vibrational analysis of the DFT-optimized supramolecular structure indicates that each pair of $\mathrm{O}-\mathrm{H}$ groups in the network present two distinct stretching modes (i.e., one symmetric with a lower IR intensity signal and one anti-symmetric with higher intensity) with the symmetric stretching of the hydrogens involved in the shorter $\mathrm{H}$-bonds characterized by the lowest vibrational frequency of $3501 \mathrm{~cm}^{-1}$ (Figure S1). The same analysis of one isolated BCF or its 1:1 complex with nitromethane shows that the corresponding $\mathrm{O}-\mathrm{H}$ stretching modes have vibrational frequencies of 3693 and $3670 \mathrm{~cm}^{-1}$, respectively (Figures S2 and S3). Therefore, the formation of a tetrameric adduct with nitromethane results in a redshift of the $\mathrm{O}-\mathrm{H}$ stretching frequency as large as $192 \mathrm{~cm}^{-1}$ relative to isolated BCF and $169 \mathrm{~cm}^{-1}$ relative to the 1:1 complex. Since an $\mathrm{O}-\mathrm{H}$ stretching frequency reduction was shown to provide a measure of the strength of a

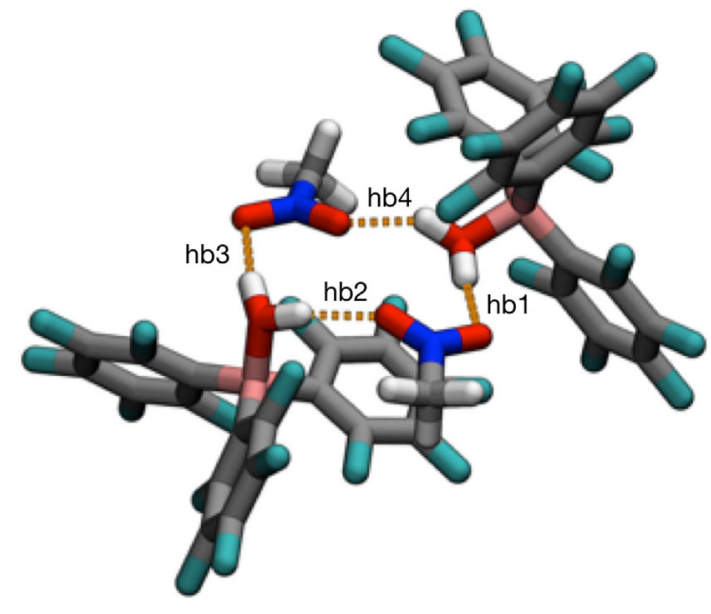

Figure 2. DFT-optimized structure for the 2:2 tetrameric self-assembly of BCF and nitromethane. The calculated structure highlights the existence of two sets of non-equivalent $\mathrm{H}$-bonds, two shorter ones ( $\mathrm{hb} 1 \mathrm{and} \mathrm{hb3}$ ) and two longer ones (hb2 and hb4).

Brønsted acid in substituted $p$ - and $m$-phenols (i.e., the lower the frequency, the stronger the acid), ${ }^{[12]}$ our calculations suggest that the 2:2 BCF/nitromethane self-assembly increases the acidity of $B C F$, in particular for the hydrogens involved in the shorter hydrogen bonds.

This counterintuitive result implies that aggregation of multiple acid molecules templated by very weak $\mathrm{H}$-bond acceptors may have the net effect of increasing the acidity, and hence catalytic activity, of the aggregate relative to the single acid molecules. Following the same modeling procedure, 3D structures of the tetrameric assembly of BCF with a variety of nitro compounds including 4-nitrobenzotrifluoride, nitrobenzene, 4nitroanisole, 2-methyl-2-nitropropane and 1-nitrohexane were generated; these compounds were previously shown to modulate the co-catalytic activity of $\mathrm{BCF} .{ }^{[6]}$ Upon geometry optimization at the DFT level of theory, the rectangular hydrogen-bonding network made of pairs of non-equivalent $\mathrm{H}$-bonds is largely preserved in all complexes, with minor distortions in some cases; see Figure S5. Vibrational analysis of the model complexes shows the same characteristic redshift in the $\mathrm{O}-\mathrm{H}$ stretching frequency with a magnitude that is dependent on the chemical nature of the nitro compound; see Table S1. Remarkably, the red shift in the anti-symmetric stretching of the short $\mathrm{H}$-bonds was found to be strongly correlated with the experimental Log(rate) with a determination coefficient $\left(R^{2}\right)$ of 0.81 (Figure 3, filled circles). This observation suggests that the $\mathrm{O}-\mathrm{H}$ stretching frequencies predicted by DFT in the 2:2 aggregate in vacuum can be used as predictors for the kinetic rate of dehydroazidation catalyzed by BCF. The same calculations in benzene for a subset of compounds using an implicit-solvent model indicate that solvation effects in nonpolar media play only a minor role on the structure and the $\mathrm{O}-\mathrm{H}$ stretching frequency of the 2:2 assembly, thus justifying the use of gas phase calculations; see Supporting Information. In addition, an analysis of the level of theory on the quantification of the $\mathrm{O}-\mathrm{H}$ stretching frequency shift upon self-assembly indicates that the use of the $6-31 G(d, p)$ basis set provides reliable predictions 
<smiles>COc1ccc([N+](=O)[O-])cc1</smiles>

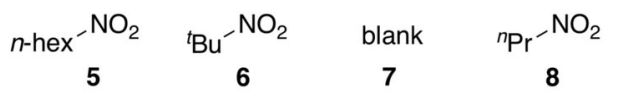

$$
\mathrm{Pr}^{-}-\mathrm{NO}_{2} \mathrm{Me} \smile \mathrm{NO}_{2}
$$

9

10<smiles>COc1ccccc1[N+](=O)[O-]</smiles>

11

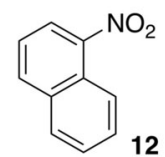

Figure 3. Correlation of the experimental kinetic rate with the DFT calculated frequency of the anti-symmetric stretching of the short $\mathrm{H}$-bonds in the 2:2 aggregate. Filled and empty data points correspond to the training (six data points + blank) and test (five data points) sets of nitro compounds investigated here.

at the lowest computational effort; see Supporting Information.

To evaluate the statistical significance of the correlation in Figure 3, two validation schemes were followed: ${ }^{[13]}$ a cross internal validation based on a leave-one-out (LOO) analysis; and an external validation based on a new set of five nitro compounds (1-nitropropane, 2-nitropropane, nitroethane, 2-nitroanisole and 1-nitronaphtalene), whose influence on the reaction rate was previously unknown and determined by GC-MS; see Supporting Information. The internal validation indicates that the DFT model presents a positive predictive profile with a cross-validated squared correlation coefficient $\left(q^{2}\right)$ of 0.46 (Table S3). The external validation shows that kinetic rates predicted by the DFT model are strongly correlated with the experimental rates with $R^{2}$ of 0.70 ; see Figure 3 (empty circles). In addition, the statistical parameters of the DFT model appear to be robust to randomization in alternative training/test sets, which is consistent with the absence of random correlations (Table S4). The predictive character of the DFT model for the experimental Log(rate) supports the assertion that tetrameric self-assembly of BCF with nitro compounds is a critical feature of the catalytic mechanism in the azidation of tertiary alcohols. ${ }^{[6]}$

A similar model for the Log(rate) was constructed using experimentally observed IR stretches, that is, the kinetic rate of azidation was correlated with the frequency at the maximum of the $\mathrm{OH}$-stretching band in the experimental IR spectrum; see Supporting Information. Although this IR model was predictive for the initial set of nitro compounds, it was not for the test set (Figure S7). In particular, the accelerations produced by 2-nitroanisole and 1-nitronapthalene (entries 11-12 in Table S1) are reversed by the IR model, which produces an anti-correlation between predicted and observed Log(rate) in the external validation; see Figure S8. The more limited predictive power of the IR model compared to DFT is explained on the basis that the intense $\mathrm{O}-\mathrm{H}$ stretching bands derive from an overlap of multiple stretching modes, which limits the resolution of the experimental determination. Despite possible inaccuracies associated with the DFT functional/basis set, ${ }^{[14]}$ the computational vibrational analysis uniquely allows to quantify the red shift in the anti-symmetric stretching of the short $\mathrm{H}$-bonds, which is predictive for the Log(rate) (Figure 3).

The formation of the catalytically active species isolated by calculations involves a largely unfavorable entropic penalty due to the association of four molecular entities. To test the thermodynamic stability of the 2:2 assemblies, the free energy of association was investigated in the rigid-rotor harmonic oscillator (RRHO) approximation; see Supporting Information. The results show that all 2:2 complexes involving nitro compounds are marginally stable with standard association free energies ranging from +3 to $+14 \mathrm{kcal} \mathrm{mol}^{-1}$ (Table S7). Although the 2:2 complexes are predicted as rarely populated in solution, we reason that if the self-assembly were faster than the background reaction, one would still observe a co-catalytic effect. Based on this hypothesis and using the free energy of self-assembly as a lower-bound estimate for the kinetic barrier of (tetrameric) association, we evaluated the activation free energy of the background reaction using Eyring's equation. ${ }^{[15]}$ The results show that in the absence of nitro compounds the activation barrier for dehydroazidation is at least $10 \mathrm{kcal} \mathrm{mol}^{-1}$ higher than the kinetic barrier for self-assembly. We suggest that albeit rarely populated, 2:2 self-assembly may have a detectable effect on the dehydroazidation rate.

Last, the DFT model was used to explore new chemotypes capable of playing the same co-catalytic role as nitro compounds. For this purpose, six representative compounds were selected on the basis of their ability to weakly accept hydrogen bonds on two different atoms (Table 1). For each compound, the tetrameric assembly with BCF was modeled and the relevant $\mathrm{O}-\mathrm{H}$ stretching frequencies calculated by DFT. In four out of six cases, the assembly preserved a rectangularshape and a planar hydrogen-bonded network after geometry optimization. Of these four, the Log(rate) predicted by the DFT model was in good correspondence with the observed reactivity after $24 \mathrm{~h}$ under standard reaction conditions in three cases (entries 1-3). Also, the two compounds that did not result in stable tetrameric complexes in the calculations did not show any accelerating effect on the reaction (entries 5-6). Most importantly, as predicted by the DFT model, diethylsulfate (entry 1 ) as additive showed a similar accelerating effect to nitromethane, ${ }^{[16]}$ the tetrameric assembly computed for diethylsulfate is shown in Figure 4. Indeed, the method of initial rates 
Table 1. Calculated frequency analysis for tetrameric self-assembly of BCF with various promoters and comparison to the observed reactivity in the dehydroazidation of alcohols.

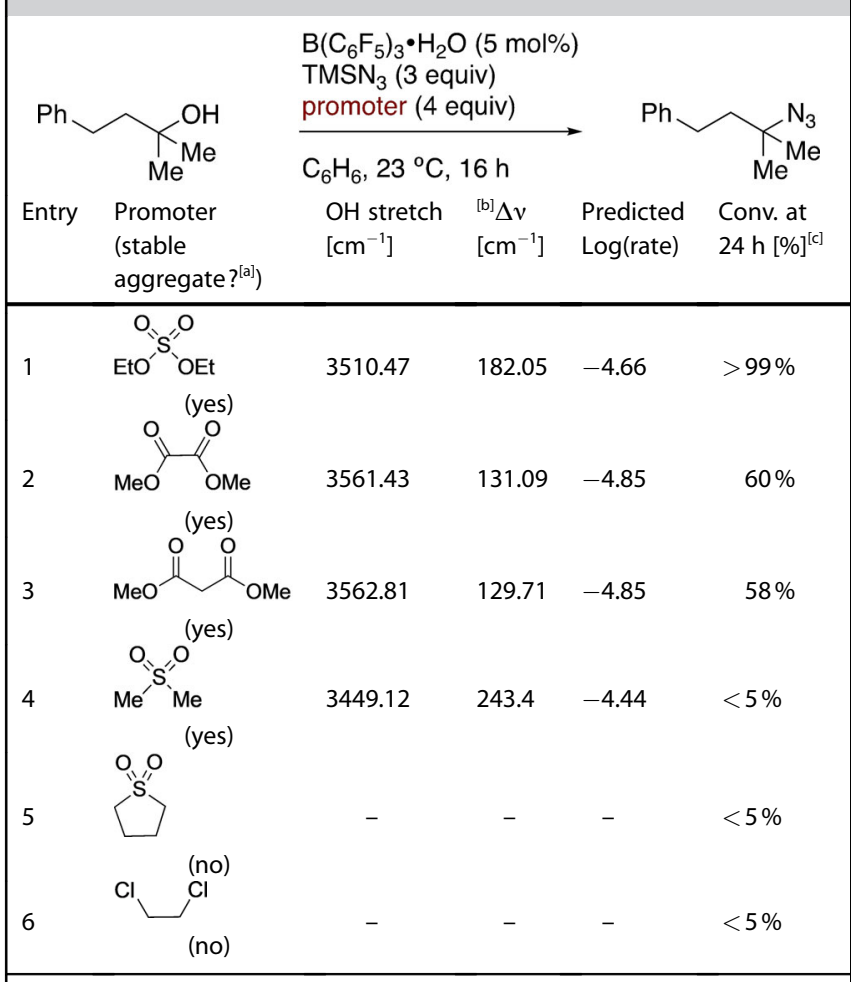

[a] A complex was considered stable if it preserves a rectangular-shape and planar hydrogen bond network as obtained in tetramer self-assembly of BCF with nitro compounds. [b] Difference between the $\mathrm{OH}$-stretching frequency of BCF in the complex and alone $\left(3692.52 \mathrm{~cm}^{-1}\right)$. [c] Reaction monitored by GCMS relative to dodecane as internal standard.

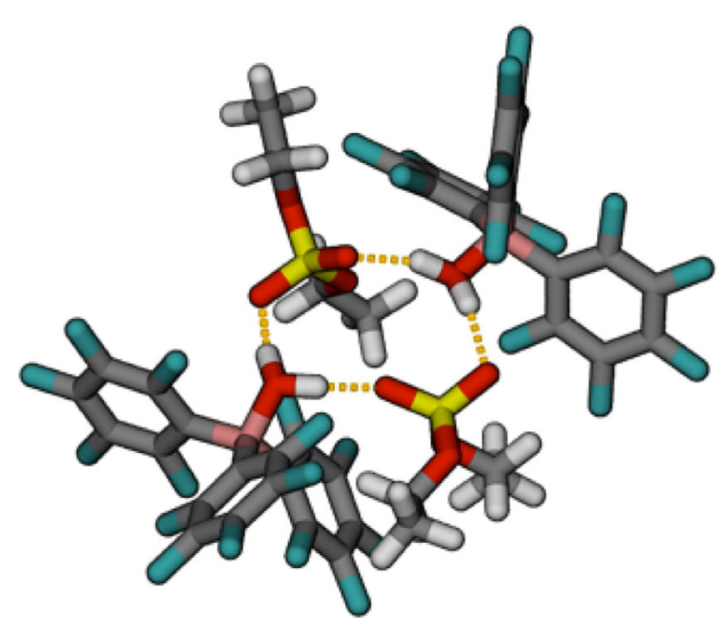

Figure 4. Optimized structure for the complex BCF/diethylsulfate (2:2).

showed a kinetic order dependence of 2.55 on diethylsulfate, consistent with higher order aggregates being involved in catalysis; see Supporting Information.

Notably, this is the first time that an additive other than a nitro compound has been shown to induce changes in the kinetic order dependence and an accelerating effect in the azi- dation reaction. The incorrect prediction for Log(rate) in entry 4 illustrates that above a certain threshold of $\mathrm{H}$-bond accepting ability, the buffering effect of the additive dominates any positive effect arising from aggregation; see Supporting Information for a discussion.

In summary, DFT modeling of 2:2 H-bonded aggregates of nitro compounds and $\mathrm{BCF}$ yields $\mathrm{O}-\mathrm{H}$ stretching frequencies that are predictive of the experimentally observed rates in reactions that use the corresponding nitro compound as promoter and can be used to guide the rational design of new co-catalysts. The structural model of the aggregate was used to identify sulfate esters as a new class of promoters, which was verified experimentally a posteriori. The correlation between experiments and calculations suggests that the increased acidity of such aggregates could indeed be responsible for the higher order kinetic concentration dependence observed in Brønsted acid catalyzed alcohol dehydroazidation. ${ }^{[6]}$ It remains to be determined whether this supramolecular mechanistic model can be extrapolated to other reactions shown to have higher order dependence on Brønsted acids when nitro compounds are present, such as olefin hydrochlorination. ${ }^{[4]}$ More broadly, the important implication of this model is that a deep understanding of Brønsted acid catalysis requires consideration of not only the molecular structure and $\mathrm{p} K_{\mathrm{a}}$ of the Brønsted acid or the bulk properties of the solvent, but also the microscopic nature of the supramolecular environment that takes into account the weak interactions between all molecules in the reaction mixture.

\section{Acknowledgements}

The authors are grateful to Chris Rowley, Vincent Robert and Antonio Monari for useful discussions. This work was supported in part by the Agence National de la Recherche through the LabEx "Chemistry of Complex Systems" (ANR-10-LABX-0026 CSC; CSC-MCE-15). Financial support from the International Center for Frontier Research in Chemistry (icFRC) is gratefully acknowledged.

\section{Conflict of interest}

The authors declare no conflict of interest.

Keywords: Brønsted acid catalysis - H-bond aggregation molecular modeling - nitro compounds - rational de novo design

[1] M. Eigen, Angew. Chem. Int. Ed. Engl. 1964, 3, 1-19; Angew. Chem. 1963, 75, 489-508.

[2] a) Z. Tian, A. Fattahi, L. Lis, S. R. Kass, J. Am. Chem. Soc. 2009, 131, 16984-16988; b) E. V. Beletskiy, J. Schmidt, X.-B. Wang, S. R. Kass, J. Am. Chem. Soc. 2012, 134, 18534-18537; c) A. Shokri, X.-B. Wang, S. R. Kass, J. Am. Chem. Soc. 2013, 135, 9525-9530; d) A. Shokri, Y. Wang, G. A. O'Doherty, X.-B. Wang, S. R. Kass, J. Am. Chem. Soc. 2013, 135, 1791917924 ; e) P. M. Pihko in Hydrogen Bonding in Organic Synthesis. WileyVCH, Weinheim, 2009; f) H. Yamamoto, K. Futatsugi, Angew. Chem. Int. Ed. 2005, 44, 1924-1942; Angew. Chem. 2005, 117, 1958-1977; g) A. 
Warshel, P. K. Sharma, M. Kato, Y. Ziang, H. Liu, M. H. M. Olsson, Chem. Rev. 2006, 106, 3210-3235.

[3] a) M. R. Monaco, B. Poladura, M. Diaz de los Bernardos, M. Leutzsch, R. Goddard, B. List, Angew. Chem. Int. Ed. 2014, 53, 7063-7067; Angew. Chem. 2014, 126, 7183-7187; b) M. R. Monaco, S. Prévost, B. List Angew. Chem. Int. Ed. 2014, 53, 8142-8145; Angew. Chem. 2014, 126, $8280-8283$; c) M. R. Monaco, S. Prévost, B. List, J. Am. Chem. Soc. 2014 136, $16982-16985$; d) S. Liao, M. Leutzsch, M. R. Monaco, B. List, J. Am. Chem. Soc. 2016, 138, 5230-5233; e) M. R. Monaco, D. Fazzi, N. Tsuji, M. Leutzsch, S. Liao, W. Thiel, B. List, J. Am. Chem. Soc. 2016, 138, $14740-$ 14749; f) A. Berkessel, J. A. Adrio, Adv. Synth. Catal. 2004, 346, 275-280; g) A. Berkessel, J. A. Adrio, D. Hüttenhain, J. M. Neudörfl, J. Am. Chem. Soc. 2006, 128, 8421-8426; h) A. Berkessel, J. A. Adrio, J. Am. Chem. Soc. 2006, 128, 13412-13420.

[4] a) Y. Pocker, J. Chem. Soc. 1960, $1292-1297$; b) Y. Pocker, K. D. Stevens, J. J. Champoux, J. Am. Chem. Soc. 1969, 91, 4199-4205; c) Y. Pocker, K. D. Stevens, J. Am. Chem. Soc. 1969, 91, 4205-4210.

[5] Some have attributed the unusual kinetic order to the polarity of the bulk solvent, though no explanation is given as to why other polar solvents do not induce a similar effect, see: G. B. Sergeev, V. V. Smirnov, T. N. Rostovshchikova, Russ. Chem. Rev. 1983, 52, 259-274 and references therein.

[6] M. Dryzhakov, M. Hellal, E. Wolf, F. C. Falk, J. Moran, J. Am. Chem. Soc. 2015, 137, 9555-9558.

[7] $\mathrm{B}\left(\mathrm{C}_{6} \mathrm{~F}_{5}\right)_{3} \cdot \mathrm{H}_{2} \mathrm{O}$ has a $\mathrm{p} K_{\mathrm{a}}$ of 8.4 in $\mathrm{MeCN}$ and has been studied in detail. a) A. A. Danopoulos, J. R. Galsworthy, M. L. H. Green, S. Cafferkey, L. H. Doerrer, M. B. Hursthouse, Chem. Commun. 1998, 2529-2530; b) C. Bergquist, B. M. Bridgewater, C. J. Harlan, J. R. Norton, R. A. Friesner, G. Parkin, J. Am. Chem. Soc. 2000, 122, 10581-10590; c) T. Beringhelli, D. Maggioni, G. D'Alfonso, Organometallics 2001, 20, 4927-4938; d) A. Di Saverio, F. Focante, I. Camurati, L. Resconi, T. Beringhelli, G. D'Alfonso, D. Donghi, D. Maggioni, P. Mercandelli, A. Sironi, Inorg. Chem. 2005, 44, $5030-5041$.

[8] F. T. Schevenels, M. Shen, S. A. Snyder, J. Am. Chem. Soc. 2017, 139, 6329-6337.

[9] a) W. F. Baitinger, P. v. R. Schleyer, T. S. S. R. Murty, L. Robinson, Tetrahedron 1964, 20, 1635-1647; b) H. E. Ungnade, E. M. Roberts, L. W. Kis- singer, J. Phys. Chem. 1964, 68, 3225 - 3228; c) M. C. Etter, Z. UrbanczykLipkowska, M. Zia-Ebrahimi, T. W. Panuto, J. Am. Chem. Soc. 1990, 112, 8415-8426; d) C. Laurence, M. Berthelot, M. Lucon, D. G. Morris, J. Chem. Soc. Perkin Trans. 2 1994, 491-493. For a seminal example of dual hydrogen bond catalysis of a nitroalkene, see: e) T. Okino, Y. Hoashi, Y. Takemoto, J. Am. Chem. Soc. 2003, 125, 12672-12673.

[10] R. Sure, S. Grimme, J. Comput. Chem. 2013, 34, 1672-1685.

[11] J.-D. Chai, M. Head-Gordon, Phys. Chem. Chem. Phys. 2008, 10, 66156620.

[12] M. Samet, J. Buhle, Y. Zhou, S. R. Kass, J. Am. Chem. Soc. 2015, 137, $4678-4680$.

[13] a) A. Golbraikh, A. Tropsha, J. Mol. Graph. Model 2002, 20, 269-276; b) A. Golbraikh, M. Shen, Z. Xiao, Y.-D. Xiao, K.-H. Lee, A. Tropsha, J. Comput. Aided Mol. Des. 2003, 17, 241-253.

[14] M. Thomas, M. Brehm, R. Fligg, P. Vöhringer, B. Kirchner, Phys. Chem. Chem. Phys. 2013, 15, 6608-6622.

[15] The activation free energy for the background reaction, that is, dehydroazidation in the absence of nitro compounds, was estimated from the experimental $\log \left(\right.$ rate) via Eyring's equation $k=\left(k_{B} T / h\right) \cdot \exp \left(-\Delta G^{\circ \neq} /\right.$ $\mathrm{RT}$ ). Using $\log (k)=-5.2$ (compound 7 in Table S1), Eyring's formulation predicts an activation free energy at $298 \mathrm{~K}$ and $1 \mathrm{M}$ concentration $\left(\Delta G^{\circ}\right)$ of $24.6 \mathrm{kcal} \mathrm{mol}^{-1}$, which is $10 \mathrm{kcal} \mathrm{mol}^{-1}$ higher than the free energy change on self-assembly $\left(\Delta G^{\circ}\right)$ for the least stable $2: 2$ complex (1-nitropropane in Table S7).

[16] Several control experiments ruled out the possibility that diethylsulfate acted as a stoichiometric activating agent or that trace acidic impurities were responsible for catalysis. In situ NMR experiments revealed that diethylsulfate was not consumed during the reaction and does not operate as a stoichiometric activating agent. Diethylsulfate in the absence of BCF did not catalyze the reaction.

Manuscript received: January 22, 2020

Accepted manuscript online: May 4, 2020

Version of record online: $\mathbf{\square} \mathbf{~ I L}, 0000$ 


\section{COMMUNICATION}

\section{Catalysis}

J. J. Montalvo-Acosta, M. Dryzhakov, E. Richmond, M. Cecchini, J. Moran*

\section{$\mathbf{\square}-\mathbf{\square}$}

IEg A Supramolecular Model for the CoCatalytic Role of Nitro Compounds in Brønsted Acid Catalyzed Reactions

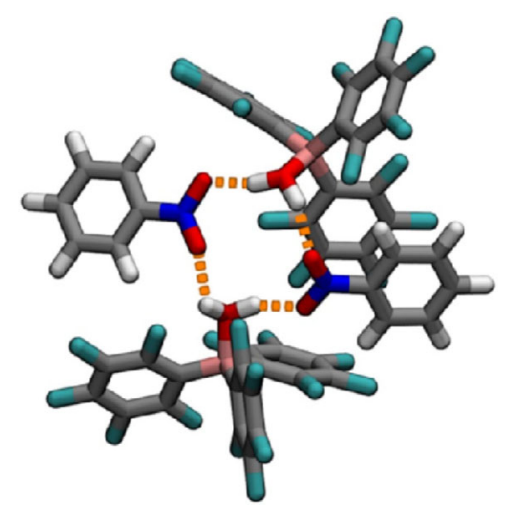

Catalysis by aggregates could explain the unusual higher order kinetics in certain Brønsted acid catalyzed reactions. Computed IR stretches of the aggregate predict reaction rates and new classes of co-catalysts (sulfate esters). 